\title{
The impact of testing and treatment on the dynamics of
}

\section{Hepatitis B virus [version 1; peer review: 2 approved]}

\section{Olajumoke Oludoun (iD1, Olukayode Adebimpe², James Ndako (D)3, Michael Adeniyi (iD4, Oluwakemi E. Abiodun (D) 5 , Babatunde Gbadamosi ${ }^{6}$}

\author{
${ }^{1}$ Physical sciences, Landmark University, Omu Aran, Kwara State, Nigeria \\ ${ }^{2}$ Physical Sciences, Landmark University, Omu Aran, Kwara, Nigeria \\ ${ }^{3}$ Biological Sciences, Landmark University, Omu Aran, Kwara, Nigeria \\ ${ }^{4}$ Mathematics, Lagos State Polytechnic, Lagos, Lagos, Nigeria \\ ${ }^{5}$ Physical Sciences (Mathematics Programme), Landmark University, Omu Aran, Kwara, Nigeria \\ ${ }^{6}$ Computer Science, Landmark University, Omu Aran, Kwara, Nigeria
}

V1 First published: 17 Sep 2021, 10:936

https://doi.org/10.12688/f1000research.72865.1

Latest published: 17 Sep 2021, 10:936

https://doi.org/10.12688/f1000research.72865.1

\section{Abstract}

Despite the intervention of WHO on vaccination for reducing the spread of Hepatitis B Virus (HBV), there are records of the high prevalence of $\mathrm{HBV}$ in some regions. In this paper, a mathematical model was formulated to analyze the acquisition and transmission process of the virus with the view of identifying the possible way of reducing the menace and mitigating the risk of the virus. The models' positivity and boundedness were demonstrated using well-known theorems. Equating the differential equations to zero demonstrates the equilibria of the solutions i.e., the disease-free and endemic equilibrium. The next Generation Matrix method was used to compute the basic reproduction number for the models. Local and global stabilities of the models were shown via linearization and Lyapunov function methods respectively. The importance of testing and treatment on the dynamics of HBV were fully discussed in this paper. It was discovered that testing at the acute stage of the virus and chronic unaware state helps in better management of the virus.

\section{Keywords}

: Positivity and boundedness of solutions, Equilibria of solutions, Next generation matrix, Linearization, Lyapunov functions, local and global stabilities.

\section{Open Peer Review

\begin{tabular}{lcc} 
Approval Status & 1 & 2 \\
\hline version 1 & & \\
17 Sep 2021 & view & view \\
\hline
\end{tabular}

1. Sulyman Olakunle Salawu, Landmark University, Omu-aran, Nigeria

2. Segun oke ID, University of Pretoria,

Pretoria, South Africa

Any reports and responses or comments on the article can be found at the end of the article. 
Corresponding author: Olajumoke Oludoun (oludoun.olajumoke@Imu.edu.ng)

Author roles: Oludoun O: Conceptualization, Methodology, Writing - Original Draft Preparation; Adebimpe O: Conceptualization, Writing - Review \& Editing; Ndako J: Supervision, Writing - Review \& Editing; Adeniyi M: Methodology; Abiodun OE: Methodology, Writing - Original Draft Preparation; Gbadamosi B: Formal Analysis

Competing interests: No competing interests were disclosed.

Grant information: The author(s) declared that no grants were involved in supporting this work.

Copyright: $\odot 2021$ Oludoun $O$ et al. This is an open access article distributed under the terms of the Creative Commons Attribution License, which permits unrestricted use, distribution, and reproduction in any medium, provided the original work is properly cited.

How to cite this article: Oludoun $O$, Adebimpe O, Ndako J et al. The impact of testing and treatment on the dynamics of Hepatitis B virus [version 1; peer review: 2 approved] F1000Research 2021, 10:936 https://doi.org/10.12688/f1000research.72865.1

First published: 17 Sep 2021, 10:936 https://doi.org/10.12688/f1000research.72865.1 


\section{Introduction}

Hepatitis is an inflammation/scarring of the liver that contributes to various health complications, including death. It occurs due to an immune system attack by the virus in the liver and damages this vital organ of the body in the process (Ciupe et al., 2014). The hepatitis B virus (HBV) can survive outside the body for at least seven days. If the virus enters the body of someone who is not protected by vaccination during this time, it can still cause infection. The average incubation time for $\mathrm{HBV}$ is 75 days, but it can range from 30 to 180 days. Within 30 to 60 days of infection, the virus may be detected, persist, and grow into chronic hepatitis B (CDC, 2019). Hepatitis B is most common in the Western Pacific region with prevalence rate $6.2 \%$ and Africa with prevalence rate of $6.1 \%$, with the Americas region $(0.7 \%)$ having the lowest prevalence (WHO, 2019).

In highly endemic areas, the most common form of transmission of hepatitis B is from mother to child at birth (vertical transmission) or through horizontal route (contact with infected blood), particularly from infected children to uninfected children during the first five years of life. Chronic infection develops in infants infected by their mothers or before the age of five. It is often transmitted through transdermal or mucosal contact of infected persons to infected blood and different body fluids, such as spittle, catamenial, vaginal and spermatic fluids and, to a lesser degree, perspiration, breast milk, tears, and urine. In particular, hepatitis B can be transmitted through sexual contact in unvaccinated men who have sex with men (MSM) and heterosexual people who have multiple sexual partners or have contact with sex workers. However, adult infection contributes to chronic hepatitis in less than $5 \%$ of cases. This transmission may similarly ensue when needles and syringes are reused, whether in healthcare settings or among drug users. Furthermore, an infection can occur during medical, surgical, and dental procedures, such as tattooing or using razors and other similar objects contaminated with infected blood (Mpeshe and Nyerere, 2019).

\section{Mathematical formulation}

Some chronic carriers are unaware of their status and as such transmit the virus unknowingly and also at higher risk of cirrhosis and makes treatment less effective (Niederau, 2014, Mcpherson et al., 2013, Cohen et al., 2011, Piorkowsky, 2009, Lin et al., 2009, Meffre et al., 2004).

In view of this, this model is developed to factor the aforementioned set of people. In the model, the population is divided into the following different groups: the susceptible, the acute, the chronic unaware carriers, the chronic aware carriers, the treated chronic aware and the recovered individuals.

The total population at time $t$, denoted by $N(t)$ is divided into the six subgroups corresponding to different epidemiological status: susceptible individuals $S(t)$, acute $A(t)$, unaware chronically infected $C_{u}(t)$, aware chronically infected $C_{a}(t)$, treated $T_{c}(t)$, and removed/recovered class $R(t)$. The model equation is subject to the initial conditions,

$$
S(t) \geq 0, A(t) \geq 0, C_{u}(t) \geq 0, C_{a}(t) \geq 0, T_{c}(t) \geq 0, R(t) \geq 0
$$

Figure 1 represents schematically the epidemiology of HBV infected model. The different disease stages are reproduced by the different circle and the arrows indicate the way individual progress from one stage to the other. It is assumed that at

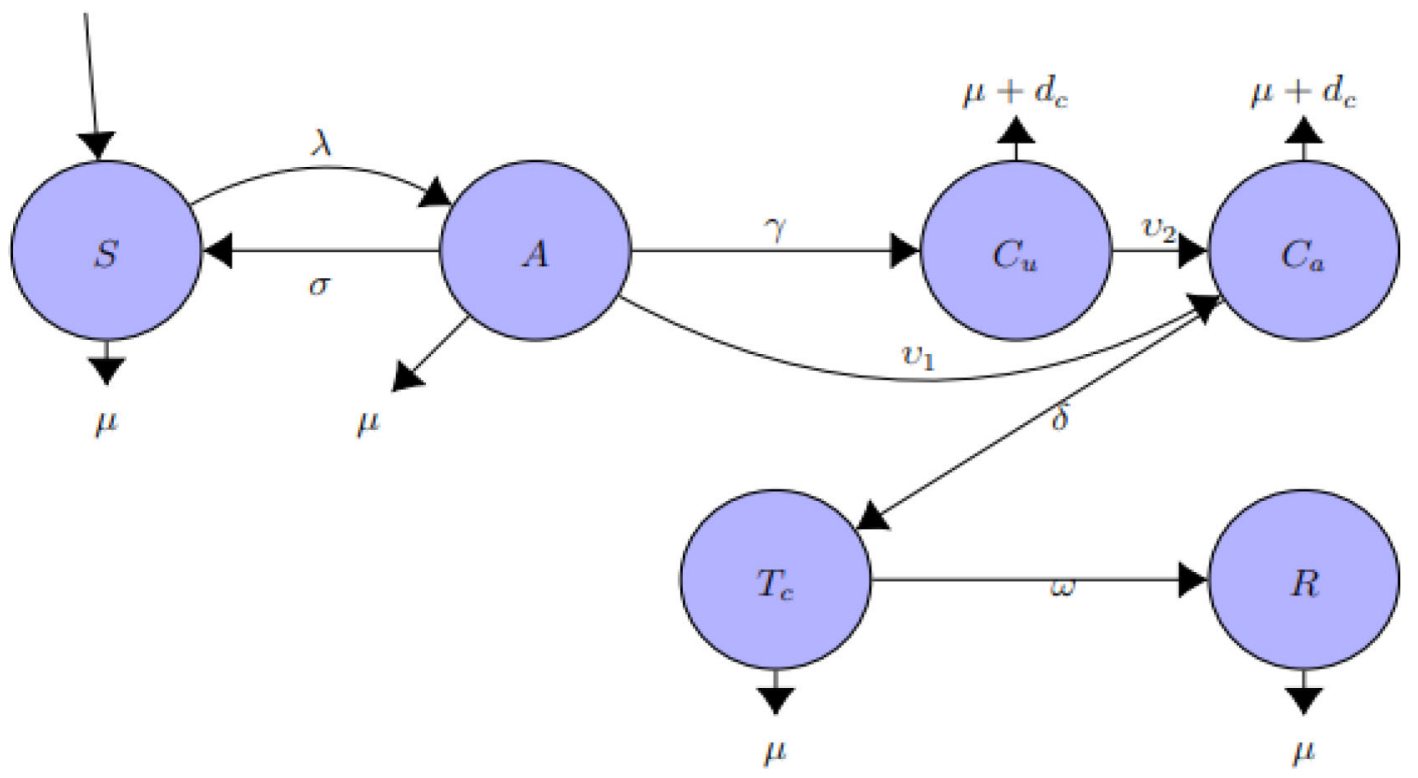

Figure 1. Compartmental flow diagram of HBV model. 
time, $t$, susceptible individuals, $S$, enter the population at a constant rate, $\Pi$. For all classes, individuals die at a constant natural mortality rate, $\mu$.HBV chronically infected individuals $\left(C_{u}(t), C_{a}(t)\right)$ have an additional death rate due to $\mathrm{HBV}$, $\mathbb{d}_{c}$ (Zhang and Zhang (2018)). It is assumed that HBV infected individuals on treatment, $T_{c}(t)$ do not transmit HBV infection. Susceptible individuals, $S(t)$, may acquire $\mathrm{HBV}$ infection when in contact with individuals in $\mathrm{A}, C_{u}$, and $C_{a}$, populace at a rate, $\lambda$ (force of infection associated with $\mathrm{HBV}$ ), where

$$
\lambda=\frac{\beta\left(A+\alpha_{1} C_{u}+\alpha_{2} C_{a}\right)}{N}
$$

Parameter $\beta$ represents the probability that a contact will result in an HBV infection while $\alpha_{1}, \alpha_{2}>1$ respectively account for modification parameter of chronic HBV-infected individuals.

A proportion of the acute $\mathrm{HBV}$-infected individuals, $\sigma$, spontaneously clear the virus, then return to being susceptible. The HBV acutely infected individuals develop to chronic without been aware if no testing at a rate, $\gamma$. The acutely infected and chronic unaware individual progress to chronic aware stage with a testing $v_{1}, v_{2}$ respectively and moved to treatment stage after testing at the rate $\delta . \omega$ is the recovery rate of treated infected individual with full immunity.

These assumptions lead to the system of equations in (3)

$$
\begin{aligned}
\frac{d S}{d t} & =\Pi-\lambda S+\sigma A-\mu S \\
\frac{d A}{d t} & =\lambda S-\left(\sigma+\gamma+v_{1}\right) A \\
\frac{d C_{u}}{d t} & =\gamma A-\left(v_{2}+\mu+d_{c}\right) C_{u} \\
\frac{d C_{a}}{d t} & =v_{2} C_{u}+v_{1} A-\left(\delta+\mu+d_{c}\right) C_{a} \\
\frac{d T_{c}}{d t} & =\delta C_{a}-(\omega+\mu) T_{c} \\
\frac{d R}{d t} & =\omega T_{c}-\mu R
\end{aligned}
$$

where $\lambda=\frac{\beta\left(A+\alpha_{1} C_{u}+\alpha_{2} C_{a}\right)}{N}$

2.1 Positivity and boundedness of solutions

For the system of equations (3) to be epidemiologically meaningful, it is important to prove that all solution with nonnegative initial conditions will remain non-negative.

Lemma 1: The initial values of the parameters are

$$
\left\{S(0) \geq 0, A(0) \geq 0, C_{u}(0) \geq 0, C_{a}(0) \geq 0, T_{c}(0) \geq 0, R(0) \geq, 0 \text { and } \mathrm{N}(0) \geq 0\right\} \in \Phi
$$

Then the solution of the model $\left\{S(t), A(t), C_{u}(t), C_{a}(t), T_{c}(t), R(t), N(t)\right\}$ is positive for all $t \geq 0$.

\section{Proof}

Considering the first equation in (3),

$$
\begin{gathered}
\frac{d S}{d t}=\Pi-\lambda S+\sigma A-\mu S \\
\frac{d S}{d t} \geq-(\lambda+\mu) \\
S \int \frac{1}{S} d S \geq \int-(\lambda+\mu) d t \\
S \geq S_{0} e^{-(\lambda+\mu) t} \geq 0
\end{gathered}
$$

Hence, $S \geq 0$ 
with respect to the second equation in (3);

$$
\begin{gathered}
\frac{d A}{d t}=\lambda S-\left(\sigma+\gamma+v_{1}\right) A \\
\frac{d A}{d t} \geq-\left(\sigma+\gamma+v_{1}\right) A \\
\int \frac{1}{A} d A \geq \int-\left(\sigma+\gamma+v_{1}\right) d t \\
A \geq A_{0} e^{-\left(\sigma+\gamma+v_{1}\right) t} \geq 0
\end{gathered}
$$

Hence, $A \geq 0$. Same goes for the other compartments

Clearly, the above state variables are positive on bounding plane $\mathbb{R}_{+}^{6}$.

For the boundedness the following calculation follows:

$$
\begin{gathered}
N(t)=S(t)+A(t)+C_{u}(t)+C_{a}(t)+T_{c}(t)+R(t) \\
N^{\prime}=S^{\prime}+A^{\prime}+C_{u}{ }^{\prime}+C_{a}{ }^{\prime}+T_{c}{ }^{\prime}+R^{\prime} \\
N^{\prime}=\Pi-\lambda S+\sigma A-\mu S+\lambda S-\left(\sigma+\gamma+v_{1}\right) A+\gamma A-\left(v_{2}+\mu+d_{c}\right) C_{u}+v_{2} C_{u}+v_{1} A \\
-\left(\delta+\mu+d_{c}\right) C_{a}+\delta C_{a}-(\omega+\mu) T_{c}+\omega T_{c}-\mu R
\end{gathered}
$$

Simplifying:

$$
\begin{gathered}
N^{\prime}+\mu N=\Pi-d_{c} C_{u} \\
N^{\prime}+\mu N \leq \Pi
\end{gathered}
$$

Integrating gives:

$$
\begin{aligned}
& N^{\prime} \leq \frac{\Pi}{\mu}+k e^{-\mu t} \\
& \max _{\lim _{n \rightarrow \infty}} N \leq \lim _{n \rightarrow \infty}\left(\frac{\Pi}{\mu}+k e^{-\mu t}\right) \leq \frac{\Pi}{\mu}
\end{aligned}
$$

It follows that the solutions of the model system (3) are positive and bounded in the region

$$
\mathcal{T}=\left\{\left(S+A+C_{u}+C_{a}+T_{c}+R\right)\right\} \in \mathbb{R}_{+}^{6}: S+A+C_{u}+C_{a}+T_{c}+R \leq \frac{\Pi}{\mu}
$$

It follows from Lemma 1 that it is sufficient to consider the dynamics of system (3) and the model can be considered to be epidemiologically well-posed.

\subsection{Equilibrium points}

The disease-free equilibrium of the equation (3) exists and is given by:

$$
\left(E_{0}\right)=\left[\frac{\Pi}{\mu}, 0,0,0,0,0\right]
$$

The endemic steady states are calculated here which is done by setting system of equation in (3.3.3) to zero and setting $S=S^{*}, A=A^{*}, C_{u}=C_{u}{ }^{*}, C_{a}=C_{a}{ }^{*}, T_{c}=T_{c}{ }^{*}, R=R^{*}$ so that 


$$
\begin{aligned}
& S^{*}=\left(\begin{array}{l}
\Pi\left(\mu^{3}+\left(\delta+\gamma+2 d_{c}+v_{1}+v_{2}\right) \mu^{2}+\left(d_{c}^{2}+\left(v_{1}+v_{2}+\delta+\gamma\right) d_{c}+\left(v_{2}+\delta\right) \gamma+\left(v_{2}+\delta\right) v_{1}+\delta v_{2}\right) \mu\right) \\
+\delta\left(v_{1} d_{c}+v_{2}\left(v_{1}+\gamma\right)\right)\left(v_{1}+\sigma+\gamma\right)
\end{array}\right) \\
& A^{*}=-\left(\begin{array}{c}
\left(v_{1}-\beta+\gamma+\sigma\right) d_{c}^{2}+\left(\left(2 v_{1}-2 \beta+2 \gamma+2 \sigma\right) \mu+\left(v_{2}+\delta-\beta \alpha_{1}\right) \gamma+\left(v_{2}+\delta-\beta \alpha_{2}\right) v_{1}-(\beta-\sigma)\left(v_{2}+\delta\right)\right) d_{c} \\
+\left(v_{1}-\beta+\gamma+\sigma\right) \mu^{2}+\left(\left(v_{2}+\delta-\beta \alpha_{1}\right) \gamma+\left(v_{2}+\delta-\beta \alpha_{2}\right) v_{1}-(\beta-\sigma)\left(v_{2}+\delta\right)\right) \mu+\left(\left(-\delta \alpha_{1}-\alpha_{2} v_{2}\right) \beta\right) \Pi \\
L
\end{array}\right) \\
& C_{u}{ }^{*}=A^{*} \gamma \\
& C_{a}{ }^{*}=\frac{C_{u}{ }^{*}\left(\left(v_{1}+v_{1} \mu\right)+v_{2}\left(v_{1}+\gamma\right)\right)}{\gamma} \\
& T_{c}{ }^{*}=-\left(C_{a}{ }^{*}\left(\frac{\mu^{2}}{d_{c}{ }^{2}}+\frac{\mu}{d_{c}}+\frac{v_{2}}{\mu^{2}}+\frac{1}{v_{1}+v_{2}}\right) \delta\right) \\
& R^{*}=\omega T_{c}{ }^{*}
\end{aligned}
$$

where

$$
\begin{aligned}
L= & \left(\left(v_{2}+\mu+d_{c}\right)\left(v_{1}+\gamma+\sigma\right) \mu^{3}+\left(\left(2 v_{1}+2 \gamma+2 \sigma\right) d_{c}\right)+\left(v_{1}+\gamma+\sigma\right) v_{2}+(\beta+\delta) \gamma+(\beta+\delta) v_{1}+\delta \sigma\right) \mu^{2} \\
& +\left(\left(v_{1}+\gamma+\sigma\right) d_{c}^{2}+\left(\left(v_{1}+\gamma+\sigma\right) v_{2}-\gamma^{2}+\left(2 \beta+\delta-\sigma-2 v_{1}\right) \gamma-v_{1}^{2}+(2 \beta+\delta-\sigma) v_{1}+\delta \sigma\right) d_{c}\right. \\
& \left.+\left((\beta+\delta) \gamma+(\beta+\delta) v_{1}+\delta \sigma\right) v_{2}+\beta\left(v_{1}+\gamma\right)\left(\gamma \alpha_{1}+\alpha_{2} v_{1}+\delta\right)\right) \mu-\left(v_{1}+\gamma\right)\left(v_{1}-\beta+\gamma+\sigma\right) d_{c}{ }^{2} \\
& +\left(-\left(v_{1}+\gamma\right)\left(v_{1}-\beta+\gamma+\sigma\right) v_{2}+\left(\beta \alpha_{1}-\delta\right) \gamma^{2}+\left(\left(-\delta+\left(\alpha_{1}+\alpha_{2}\right) \beta\right) v_{1}+\delta(\beta-\sigma)\right) \gamma+\left(\delta+\alpha_{2} v_{1}\right) \beta v_{1}\right) d_{c} \\
& \left.+\beta\left(\gamma \alpha_{1}+\alpha_{2} v_{1}+\delta\right) v_{2}+\delta \gamma \alpha_{1}\right)\left(v_{1}+\gamma\right)\left(\delta+\mu+d_{c}\right)(\mu+\omega)
\end{aligned}
$$

2.3 Basic reproduction number

The basic reproduction number $\left(R_{o}\right)$ which is the number of secondary infections caused by an infectious individual is determined by the next generation matrix which is given by $\rho\left(F V^{-1}\right)$

where:

$$
\begin{aligned}
& F=\left[\begin{array}{ccc}
\beta & \beta \alpha_{1} & \beta \alpha_{2} \\
0 & 0 & 0 \\
0 & 0 & 0
\end{array}\right] \\
& V=\left[\begin{array}{ccc}
\sigma+\gamma+v_{1} & 0 & 0 \\
-\gamma & d_{c}+\mu+v_{2} & 0 \\
-v_{1} & -v_{2} & d_{c}+\mu+\delta
\end{array}\right] \\
& V^{-1}=\left[\begin{array}{ccc}
\frac{1}{\sigma+\gamma+v_{1}} & 0 & 0 \\
\frac{\gamma}{\left(\sigma+\gamma+v_{1}\right)\left(d_{c}+\mu+v_{2}\right)} & \frac{1}{d_{c}+\mu+v_{2}} & 0 \\
\frac{\gamma v_{2}+v_{1} \mu+v_{1} d_{c}+v_{1} v_{2}}{\left(\sigma+\gamma+v_{1}\right)\left(d_{c}+\mu+v_{2}\right)\left(d_{c}+\mu+\delta\right)} & \frac{v_{2}}{\left(d_{c}+\mu+v_{2}\right)\left(d_{c}+\mu+\delta\right)} \frac{1}{\left(d_{c}+\mu+\delta\right)}
\end{array}\right]
\end{aligned}
$$




$$
R_{o}=\frac{\beta}{\sigma+\gamma+v_{1}}+\frac{\beta \alpha_{1} \gamma}{\left(\sigma+\gamma+v_{1}\right)\left(d_{c}+\mu+v_{2}\right)}+\frac{\beta \alpha_{2}\left(\gamma v_{2}+v_{1} \mu+v_{1} d_{c}+v_{1} v_{2}\right)}{\left(\sigma+\gamma+v_{1}\right)\left(d_{c}+\mu+v_{2}\right)\left(d_{c}+\mu+\delta\right)}
$$

2.4 Local stability analysis of the disease-free equilibrium $E_{0}$

Theorem 1: $E_{0}$ is locally asymptotically stable if $R_{0}<1$ and unstable if $R_{0}>1$.

Proof: The resulting matrix from the linearized model is $\frac{d X}{d t}=A X$

$$
X=\left(x_{1}, x_{2}, x_{3}, x_{4}, x_{5}, x_{6}\right)^{T},\left(x_{1}, x_{2}, x_{3}, x_{4}, x_{5}, x_{6}\right) \in R_{+}^{6}, \text { and }
$$

The resulting Jacobian matrix at $E_{0}$ is

$$
J\left(E_{0}\right)=\left[\begin{array}{llllll}
-\mu-\lambda & -\beta+\sigma & -\beta \alpha_{1} & -\beta \alpha_{2} & 0 & 0 \\
0 & \beta-\sigma-\gamma-v_{1}-\lambda & \beta \alpha_{1} & \beta \alpha_{2} & 0 & 0 \\
0 & \gamma & -d_{c}-\mu-v_{2}-\lambda & 0 & 0 & 0 \\
0 & v_{1} & v_{2} & -d_{c}-\mu-\delta-\lambda & 0 & 0 \\
0 & 0 & 0 & \delta & -\omega-\mu-\lambda & 0 \\
0 & 0 & 0 & 0 & \omega & -\mu-\lambda
\end{array}\right]
$$

From (15), $\lambda_{1}=-\mu, \lambda_{2}=-\omega-\mu, \lambda_{3}=-\mu$

and the resulting quadratic equation is:

$$
\begin{aligned}
&\left(\beta-\sigma-\gamma-v_{1}-\lambda\right)\left(-d_{c}-\mu-v_{2}-\lambda\right)\left(-d_{c}-\mu-\delta-\lambda\right)-\beta \alpha_{1}\left(-d_{c}-\mu-\delta-\lambda\right) \gamma+\beta \alpha_{2}\left(\left(-d_{c}-\mu-v_{2}-\lambda\right) v_{1}-\gamma v_{2}\right) \\
& f(\lambda)= \lambda^{3}+\left(2 \mu+v_{1}+v_{2}-\beta+\delta+\gamma+\sigma+2 d_{c}\right) \lambda^{2}+\left(\beta \alpha_{2} v_{1}-\beta \delta-2 \beta \mu-2 \beta d_{c}-\beta v_{2}+\delta \gamma+\delta \mu+\delta \sigma+\delta d_{c}+\delta v_{1}\right. \\
&\left.+\delta v_{2}+2 \gamma \mu+2 \gamma d_{c}+\gamma v_{2}+\mu^{2}+2 \mu \sigma+2 \mu d_{c}+2 \mu v_{1}+\mu v_{2}+2 \sigma d_{c}+\sigma v_{2}+d_{c}{ }^{2}+2 d_{c} v_{1}+d_{c} v_{2}+v_{1} v_{2}-\gamma \beta \alpha_{1}\right) \lambda \\
&+\gamma \mu^{2}+\gamma d_{c}{ }^{2}+\mu^{2} \sigma+\mu^{2} v_{1}+\sigma d_{c}{ }^{2}+d_{c}{ }^{2} v_{1}+\mu \sigma v_{2}+2 \mu d_{c} v_{1}+\mu v_{1} v_{2}+\sigma d_{c} v_{2}+d_{c} v_{1} v_{2}-\beta \delta \mu-\beta \delta d_{c}-\beta \delta v_{2} \\
&-2 \beta \mu d_{c}-\beta \mu v_{2}-\beta d_{c} v_{2}-\beta \mu^{2}-\beta d_{c}{ }^{2}+\delta \gamma \mu+\delta \gamma d_{c}+\delta \gamma v_{2}+\delta \mu \sigma+\delta \mu v_{1}+\delta \sigma d_{c}+\delta \sigma v_{2}+\delta d_{c} v_{1}+\delta v_{1} v_{2} \\
&+2 \gamma \mu d_{c}+\gamma \mu v_{2}+\gamma d_{c} v_{2}+2 \mu \sigma d_{c}-\beta \delta \gamma \alpha_{1}-\beta \gamma \mu \alpha_{1}-\beta \gamma \alpha_{1} d_{c}+v_{2} \gamma \beta \alpha_{2}+v_{1} \beta \alpha_{2} d_{c}+v_{1} \beta \alpha_{2} \mu+v_{1} \beta \alpha_{2} v_{2}
\end{aligned}
$$

Now, $\lambda_{1}, \lambda_{2}, \lambda_{3}<0$ since the values are assumed positive. If $R_{0}<1, E_{0}$ is stable and unstable when $R_{0}<1$.

2.5 Global stability of the disease-free equilibrium

The global behavior of the equilibrium system (3) is analyzed here in this section.

Theorem 2: For system (3), the disease-free equilibrium $E_{0}$ is asymptotically stable globally if $R_{0}<1$.

Proof: Considering the Lyapunov function defined as:

$$
\begin{gathered}
G\left(A, C_{u}, C_{a}\right)=\left(\frac{1}{B_{0}}\right) A+\left(\frac{\beta \alpha_{1}}{B_{0} B_{1}}+\frac{\beta \alpha_{2} v_{2}}{B_{0} B_{1} B_{2}}\right) C_{u}+\left(\frac{\beta \alpha_{2}}{B_{0} B_{2}}\right) C_{a} \\
G^{\prime}\left(A, C_{u}, C_{a}\right)=\left(\frac{1}{B_{0}}\right) A^{\prime}+\left(\frac{\beta \alpha_{1}}{B_{0} B_{1}}+\frac{\beta \alpha_{2} v_{2}}{B_{0} B_{1} B_{2}}\right) C_{u}{ }^{\prime}+\left(\frac{\beta \alpha_{2}}{B_{0} B_{2}}\right) C_{a}{ }^{\prime} \\
G^{\prime}\left(A, C_{u}, C_{a}\right)=\left(\frac{1}{B_{0}}\right)\left(\left(\frac{\beta\left(A+\alpha_{1} C_{u}+\alpha_{2} C_{a}\right)}{N}\right) S-\left(\sigma+\gamma+v_{1}\right) A\right)+\left(\frac{\beta \alpha_{1}}{B_{0} B_{1}}+\frac{\beta \alpha_{2} v_{2}}{B_{0} B_{1} B_{2}}\right)\left(\gamma A-\left(v_{2}+\mu+d_{c}\right) C_{u}\right) \\
+\left(\frac{\beta \alpha_{2}}{B_{0} B_{2}}\right)\left(v_{2} C_{u}+v_{1} A-\left(\delta+\mu+d_{c}\right) C_{a}\right)
\end{gathered}
$$


At DFE, $\mathrm{S}=\mathrm{N}$ so that $(20)$ becomes:

$$
\begin{aligned}
G^{\prime}\left(A, C_{u}, C_{a}\right)= & \left(\frac{1}{B_{0}}\right)\left(\beta\left(A+\alpha_{1} C_{u}+\alpha_{2} C_{a}\right)-\left(\sigma+\gamma+v_{1}\right) A\right)+\left(\frac{\beta \alpha_{1}}{B_{0} B_{1}}+\frac{\beta \alpha_{2} v_{2}}{B_{0} B_{1} B_{2}}\right)\left(\gamma A-\left(v_{2}+\mu+d_{c}\right) C_{u}\right) \\
& +\left(\frac{\beta \alpha_{2}}{B_{0} B_{2}}\right)\left(v_{2} C_{u}+v_{1} A-\left(\delta+\mu+d_{c}\right) C_{a}\right)
\end{aligned}
$$

Expanding and simplifying (21) gives:

$$
\begin{gathered}
G^{\prime}=\left[\frac{\beta}{B_{0}}+\frac{\beta \alpha_{1} \gamma}{B_{0} B_{1}}+\frac{\beta \alpha_{2} v_{2} \gamma}{B_{0} B_{1} B_{2}}+\frac{\beta \alpha_{2} v_{1}}{B_{0} B_{2}}-1\right] \boldsymbol{A}+\left[\frac{\beta \alpha_{1}}{B_{0}}-\frac{\beta \alpha_{1} B_{1}}{B_{0} B_{1}}-\frac{\beta \alpha_{2} v_{2} B_{1}}{B_{0} B_{1} B_{2}}+\frac{\beta \alpha_{2} v_{2}}{B_{0} B_{2}}\right] C_{u}+\left[\frac{\beta \alpha_{2}}{B_{0}}-\frac{\beta \alpha_{2} B_{2}}{B_{0} B_{2}}\right] C_{a} \\
G^{\prime}=\left[R_{0}-1\right] A \leq 0
\end{gathered}
$$

From Equation (23), it can be deduced that the DFE is globally stable since $R_{0}<1$.

\subsection{Local stability of endemic equilibrium}

Theorem 3: If $R_{0}>1$, then the endemic equilibrium is locally asymptotically stable.

\section{Proof:}

The endemic equilibria of system (3), denoted by $\left(S^{*}, A^{*}, C_{u}{ }^{*}, C_{a}{ }^{*}, T_{c}{ }^{*}, R^{*}\right)$, can be rewritten as:

$$
\begin{aligned}
& \text { Let } S=x+S^{*}, A=y+A^{*}, C_{u}=z+C_{u}{ }^{*}, C_{a}=h+C_{a}{ }^{*}, T_{c}=p+T_{c}{ }^{*}, R=j+R^{*} \\
& J=\left[\begin{array}{llllll}
B_{0}-\mu-\lambda & -B_{1}+\sigma & -B_{2} & -B_{3} & B_{4} & B_{5} \\
-B_{6} & B_{7}-\sigma-\gamma-v_{1}-\lambda & B_{8} & B_{9} & -B_{11} & -B_{12} \\
0 & \gamma & -d_{c}-\mu-v_{2}-\lambda & 0 & 0 & 0 \\
0 & v_{1} & v_{2} & -d_{c}-\mu-\delta-\lambda & 0 & 0 \\
0 & 0 & 0 & \delta & -\omega-\mu-\lambda & 0 \\
0 & 0 & 0 & 0 & \omega & -\mu-\lambda
\end{array}\right]
\end{aligned}
$$

From (25), $\lambda_{1}=-\mu, \lambda_{2}=-(\omega+\mu), \lambda_{3}=-\left(d_{c}+\mu+v_{2}\right)$, then;

$$
J=\left[\begin{array}{lll}
B_{0}-\mu-\lambda-B_{1}+\sigma & -B_{2} \\
-B_{6} & B_{7}-\sigma-\gamma-v_{1}-\lambda & B_{8} \\
0 & \gamma & -d_{c}-\mu-v_{2}-\lambda
\end{array}\right]
$$

from (26);

$$
\begin{aligned}
\lambda^{3} & +\left(\gamma+2 \mu+\sigma-B_{0}-B_{4}+d_{c}+v_{1}+v_{2}\right) \lambda^{2}+\left(2 \gamma \mu-B_{0} \gamma+\gamma d_{c}+\gamma v_{2}+\mu^{2}+2 \mu \sigma-\mu B_{0}-2 \mu B_{4}+\mu d_{c}+2 \mu v_{1}+\mu v_{2}\right. \\
& \left.-\sigma B_{0}+\sigma B_{3}+\sigma d_{c}+\sigma v_{2}+B_{0} B_{4}-B_{0} d_{c}-B_{0} v_{1}-B_{0} v_{2}-B_{3} B_{1}-B_{4} d_{c}-B_{4} v_{2}+d_{c} v_{1}+v_{1} v_{2}\right) \lambda+B_{5} \gamma+\gamma \mu v_{2}+\mu \sigma v_{2} \\
& +\mu v_{1} v_{2}+B_{3} B_{2} \gamma+\gamma \mu^{2}+\mu^{2} \sigma+\mu^{2} v_{1}+\mu d_{c} v_{1}+\gamma \mu d_{c}+\mu \sigma d_{c}-\mu^{2} B_{4}-\gamma \mu B_{0}-\gamma B_{0} d_{c}-\gamma B_{0} v_{2}-\mu \sigma B_{0}+\mu \sigma B_{3}+\mu B_{0} B_{4} \\
& -\mu B_{0} v_{1}-\mu B_{3} B_{1}-\mu B_{4} d_{c}-\mu B_{4} v_{2}-\sigma B_{0} d_{c}-\sigma B_{0} v_{2}+\sigma B_{3} d_{c}+\sigma B_{3} v_{2}+B_{0} B_{4} d_{c}+B_{0} B_{4} v_{2}-B_{0} d_{c} v_{1}-B_{0} v_{1} v_{2} \\
& -B_{3} B_{1} d_{c}-B_{3} B_{1} v_{2}
\end{aligned}
$$

The result of the determinant of the Jacobian matrix is of the form:

$$
a_{0} \lambda^{3}+a_{1} \lambda^{2}+a_{2} \lambda+a_{3}
$$

where

$$
\begin{gathered}
a_{0}=1 \\
a_{1}=\gamma+2 \mu+\sigma-B_{0}-B_{4}+d_{c}+v_{1}+v_{2}
\end{gathered}
$$




$$
\begin{aligned}
a_{2}= & 2 \gamma \mu-B_{0} \gamma+\gamma d_{c}+\gamma v_{2}+\mu^{2}+2 \mu \sigma-\mu B_{0}-2 \mu B_{4}+\mu d_{c}+2 \mu v_{1}+\mu v_{2}-\sigma B_{0}+\sigma B_{3}+\sigma d_{c}+\sigma v_{2}+B_{0} B_{4}-B_{0} d_{c} \\
& -B_{0} v_{1}-B_{0} v_{2}-B_{3} B_{1}-B_{4} d_{c}-B_{4} v_{2}+d_{c} v_{1}+v_{1} v_{2} \\
a_{3}= & B_{5} \gamma+\gamma \mu v_{2}+\mu \sigma v_{2}+\mu v_{1} v_{2}+B_{3} B_{2} \gamma+\gamma \mu^{2}+\mu^{2} \sigma+\mu^{2} v_{1}+\mu d_{c} v_{1}+\gamma \mu d_{c}+\mu \sigma d_{c}-\mu^{2} B_{4}-\gamma \mu B_{0}-\gamma B_{0} d_{c}-\gamma B_{0} v_{2} \\
& -\mu \sigma B_{0}+\mu \sigma B_{3}+\mu B_{0} B_{4}-\mu B_{0} v_{1}-\mu B_{3} B_{1}-\mu B_{4} d_{c}-\mu B_{4} v_{2}-\sigma B_{0} d_{c}-\sigma B_{0} v_{2}+\sigma B_{3} d_{c}+\sigma B_{3} v_{2}+B_{0} B_{4} d_{c}+B_{0} B_{4} v_{2} \\
& -B_{0} d_{c} v_{1}-B_{0} v_{1} v_{2}-B_{3} B_{1} d_{c}-B_{3} B_{1} v_{2}
\end{aligned}
$$

By the Routh-Hurwitz criterion governing the polynomials of order 3, we have the following:

1. $a_{2} . a_{3}$ are positive

2. $a_{1} a_{2}>a_{3}$

From equation (27), 1 and 2 are satisfied.

Therefore, endemic equilibrium is locally asymptotically stable.

2.7 Global stability of the endemic equilibrium

Theorem 4: The equations of the model have a positive distinctive endemic equilibrium whenever $R_{0}>1$, which is said to be globally asymptotically stable.

Proof: Considering the Lyapunov function defined as:

$$
\begin{aligned}
L\left(S^{*}, A^{*}, C_{u}{ }^{*}, C_{a}{ }^{*}, T_{c}{ }^{*}, R^{*}\right)= & \left(S-S^{*} \ln \left(\frac{S}{S^{*}}\right)\right)+\left(A-A^{*} \ln \left(\frac{A}{A^{*}}\right)\right)+\left(C_{u}-C_{u}{ }^{*} \ln \left(\frac{C_{u}}{C_{u}{ }^{*}}\right)\right) \\
& +\left(C_{a}-C_{a}{ }^{*} \ln \left(\frac{C_{a}}{C_{a}{ }^{*}}\right)\right)+\left(T_{c}-T_{c}{ }^{*} \ln \left(\frac{T_{c}}{T_{c}}\right)\right)+\left(R-R^{*} \ln \left(\frac{R}{R^{*}}\right)\right)
\end{aligned}
$$

where $L$ takes it derivative along the system directly as:

$$
\begin{aligned}
& \frac{d L}{d t}=\left(1-\frac{S^{*}}{S}\right) \frac{d S}{d t}+\left(1-\frac{A^{*}}{A}\right) \frac{d A}{d t}+\left(1-\frac{C_{u}{ }^{*}}{C_{u}}\right) \frac{d C_{u}}{d t}+\left(1-\frac{C_{a}{ }^{*}}{C_{a}}\right) \frac{d C_{a}}{d t}+\left(1-\frac{T_{c}{ }^{*}}{T_{c}}\right) \frac{d T_{c}}{d t}+\left(1-\frac{R^{*}}{R}\right) \frac{d R}{d t} \\
\frac{d L}{d t}= & \left(1-\frac{S^{*}}{S}\right)\left[\Pi-\left(\frac{\beta\left(A+\alpha_{1} C_{u}+\alpha_{2} C_{a}\right)}{N}\right) S+\sigma A-\mu S\right]+\left(1-\frac{A^{*}}{A}\right)\left[\left(\frac{\beta\left(A+\alpha_{1} C_{u}+\alpha_{2} C_{a}\right)}{N}\right) S-\left(\sigma+\gamma+v_{1}\right) A\right] \\
& +\left(1-\frac{C_{u}^{*}}{C_{u}}\right)\left[\gamma A-\left(v_{2}+\mu+d_{c}\right) C_{u}\right]+\left(1-\frac{C_{a}{ }^{*}}{C_{a}}\right)\left[v_{2} C_{u}+v_{1} A-\left(\delta+\mu+d_{c}\right) C_{a}\right]+\left(1-\frac{T_{c}{ }^{*}}{T_{c}}\right)\left[\delta C_{a}-(\omega+\mu) T_{c}\right] \\
& +\left(1-\frac{R^{*}}{R}\right)\left[\omega T_{c}-\mu R\right]
\end{aligned}
$$

At equilibrium,

$$
\begin{aligned}
\Pi & =\left(\frac{\beta\left(A *+\alpha_{1} C_{u} *+\alpha_{2} C_{a} *\right)}{N *}\right) S *-\sigma A *+\mu S * \\
\left(\sigma+\gamma+v_{1}\right) & =\left(\frac{\beta\left(A *+\alpha_{1} C_{u} *+\alpha_{2} C_{a} *\right)}{A N *}\right) S * \\
\left(v_{2}+\mu+d_{c}\right) & =\frac{\gamma A *}{C_{u} *} \\
\left(\delta+\mu+d_{c}\right) & =\frac{v_{2} C_{u} *}{C_{a} *}+\frac{\nu_{1} A *}{C_{a} *} \\
(\omega+\mu) & =\frac{\delta C_{a} *}{T_{c} *} \\
\omega & =\frac{\mu R *}{T_{c} *}
\end{aligned}
$$




$$
\begin{aligned}
& \frac{d L}{d t}=\left(1-\frac{S^{*}}{S}\right)\left[\left(\frac{\beta\left(A *+\alpha_{1} C_{u} *+\alpha_{2} C_{a} *\right)}{N *}\right) S *-\sigma A *+\mu S *-\left(\frac{\beta\left(A+\alpha_{1} C_{u}+\alpha_{2} C_{a}\right)}{N}\right) S+\sigma A-\mu S\right] \\
& +\left(1-\frac{A^{*}}{A}\right)\left[\left(\frac{\beta\left(A+\alpha_{1} C_{u}+\alpha_{2} C_{a}\right)}{N}\right) S-\left(\frac{\beta\left(A *+\alpha_{1} C_{u} *+\alpha_{2} C_{a} *\right)}{A N *}\right) S * A\right]+\left(1-\frac{C_{u}^{*}}{C_{u}}\right)\left[\gamma A-\frac{\gamma A *}{C_{u} *} C_{u}\right] \\
& +\left(1-\frac{C_{a}^{*}}{C_{a}}\right)\left[v_{2} C_{u}+v_{1} A-\frac{v_{2} C_{u^{*}}}{C_{a} *}+\frac{v_{1} A *}{C_{a} *} C_{a}\right]+\left(1-\frac{T_{c}^{*}}{T_{c}}\right)\left[\delta C_{a}-\frac{\delta C_{a} *}{T_{c} *} T_{c}\right]+\left(1-\frac{R^{*}}{R}\right)\left[\frac{\mu R *}{T_{c} *} T_{c}-\mu R\right] \\
& =\left(1-\frac{S^{*}}{S}\right)\left[\frac{\beta A * S *}{N *}+\frac{\beta \alpha_{1} C_{u} * S *}{N *}+\frac{\beta \alpha_{2} C_{a} * S *}{N *}-\sigma A *+\mu S *-\frac{\beta A S}{N}-\frac{\beta \alpha_{1} C_{u} S}{N}-\frac{\beta \alpha_{2} C_{a} S}{N}+\sigma A-\mu S\right] \\
& +\left(1-\frac{A^{*}}{A}\right)\left[\frac{\beta A S}{N}-\frac{\beta A S *}{N *}+\frac{\beta \alpha_{1} C_{u} S}{N}-\frac{\beta \alpha_{1} C_{u} * S * A}{A * N *}+\frac{\beta \alpha_{2} C_{a} S}{N}-\frac{\beta \alpha_{2} C_{a} * S * A}{A * N *}\right]+\left(1-\frac{C_{u}{ }^{*}}{C_{u}}\right) \gamma A\left[1-\frac{A * C_{u}}{A C_{u} *}\right] \\
& +\left(1-\frac{C_{a}^{*}}{C_{a}}\right)\left[v_{2} C_{u}\left(1-\frac{C_{u}{ }^{*} C_{a}}{C_{u} C_{a} *}\right)+v_{1} A\left(1-\frac{A^{*} C_{a}}{A C_{a} *}\right)\right]+\delta C_{a}\left(1-\frac{T_{c}^{*}}{T_{c}}\right)\left[1-\frac{C_{a} * T_{c}}{C_{a} T_{c} *}\right] \\
& -\mu R\left(1-\frac{R^{*}}{R}\right)\left[1-\frac{R * T_{c}}{R T_{c} *}\right] \\
& =\left(1-\frac{S^{*}}{S}\right)\left[-\frac{\beta A S}{N}\left(1-\frac{A * S * N}{A S N *}\right)-\frac{\beta \alpha_{1} C_{u} S}{N}\left(1-\frac{C_{u *} S * N}{C_{u} S N *}\right)+\frac{\beta \alpha_{2} C_{a} S}{N}\left(1-\frac{C_{a *} S * N}{C_{a} S N *}\right)-\sigma A\left(1-\frac{A^{*}}{A}\right)-\mu S-\left(1-\frac{S^{*}}{S}\right)\right] \\
& +\left(1-\frac{A^{*}}{A}\right)\left[\frac{\beta A S}{N}\left(1-\frac{A * S * N}{A S N *}\right)-\frac{\beta \alpha_{1} C_{u} S}{N}\left(1-\frac{C_{u *} S * A N}{C_{u} S A * N *}\right)+\frac{\beta \alpha_{2} C_{a} S}{N}\left(1-\frac{C_{a *} S * A N}{C_{a} S A * N *}\right)\right] \\
& +\left(1-\frac{C_{u}{ }^{*}}{C_{u}}\right) \gamma A\left[1-\frac{A * C_{u}}{A C_{u} *}\right]+\left(1-\frac{C_{a}{ }^{*}}{C_{a}}\right)\left[v_{2} C_{u}\left(1-\frac{C_{u}{ }^{*} C_{a}}{C_{u} C_{a} *}\right)+v_{1} A\left(1-\frac{A^{*} C_{a}}{A C_{a} *}\right)\right]+\delta C_{a}\left(1-\frac{T_{c}{ }^{*}}{T_{c}}\right)\left[1-\frac{C_{a} * T_{c}}{C_{a} T_{c} *}\right] \\
& -\mu R\left(1-\frac{R^{*}}{R}\right)\left[1-\frac{R * T_{c}}{R T_{c} *}\right] \\
& =-\mu S\left(1-\frac{S^{*}}{S}\right)^{2}-\frac{\beta A S}{N}\left(1-\frac{S^{*}}{S}\right)\left(1-\frac{A^{*} S^{*} N}{A S N *}\right)-\frac{\beta \alpha_{1} C_{u} S}{N}\left(1-\frac{S^{*}}{S}\right)\left(1-\frac{C_{u *} S * N}{C_{u} S N *}\right) \\
& -\frac{\beta \alpha_{2} C_{a} S}{N}\left(1-\frac{S^{*}}{S}\right)\left(1-\frac{C_{a *} S * N}{C_{a} S N *}\right)-\sigma A\left(1-\frac{S^{*}}{S}\right)\left(1-\frac{A^{*}}{A}\right)+\frac{\beta A S}{N}\left(1-\frac{A^{*}}{A}\right)\left(1-\frac{S^{*} N}{S N *}\right) \\
& +\frac{\beta \alpha_{1} C_{u} S}{N}\left(1-\frac{A^{*}}{A}\right)\left(1-\frac{C_{u *} S * \mathrm{AN}}{C_{u} S A * N *}\right)+\frac{\beta \alpha_{2} C_{a} S}{N}\left(1-\frac{A^{*}}{A}\right)\left(1-\frac{C_{a *} S * \mathrm{AN}}{C_{a} S A * N *}\right)+\sigma A\left(1-\frac{C_{u}^{*}}{C_{u}}\right)\left(1-\frac{A^{*} C_{u}}{A C_{u} *}\right) \\
& +v_{2} C_{u}\left(1-\frac{C_{a}{ }^{*}}{C_{a}}\right)\left(1-\frac{C_{u}{ }^{*} C_{a}}{C_{u} C_{a} *}\right)+v_{1} A\left(1-\frac{C_{a}{ }^{*}}{C_{a}}\right)\left(1-\frac{A^{*} C_{a}}{A C_{a} *}\right)+\delta C_{a}\left(1-\frac{T_{c}{ }^{*}}{T_{c}}\right)\left(1-\frac{C_{a} * T_{c}}{C_{a} T_{c} *}\right) \\
& -\mu R\left(1-\frac{R^{*}}{R}\right)\left(1-\frac{R * T_{c}}{R T_{c} *}\right) \\
& =-\mu S\left(1-\frac{S^{*}}{S}\right)^{2}+P_{1}\left(S, A, C_{a}, C_{u}, T_{c}, R\right)+P_{2}\left(S, A, C_{a}, C_{u}, T_{c}, R\right)
\end{aligned}
$$

where,

$$
\begin{aligned}
& P_{1}\left(S, A, C_{a}, C_{u}, T_{c}, R\right)=-\frac{\beta A S}{N}\left(1-\frac{S^{*}}{S}\right)\left(1-\frac{A^{*} S^{*} N}{A S N *}\right)-\frac{\beta \alpha_{1} C_{u} S}{N}\left(1-\frac{S^{*}}{S}\right)\left(1-\frac{C_{u *} S * N}{C_{u} S N *}\right) \\
&-\frac{\beta \alpha_{2} C_{a} S}{N}\left(1-\frac{S^{*}}{S}\right)\left(1-\frac{C_{a *} S * N}{C_{a} S N *}\right)-\sigma A\left(1-\frac{S^{*}}{S}\right)\left(1-\frac{A^{*}}{A}\right)-\mu R\left(1-\frac{R^{*}}{R}\right)\left(1-\frac{R * T_{c}}{R T_{c} *}\right) \\
& P_{2}\left(S, A, C_{a}, C_{u}, T_{c}, R\right)= \frac{\beta A S}{N}\left(1-\frac{A^{*}}{A}\right)\left(1-\frac{S^{*} N}{S N *}\right)+\frac{\beta \alpha_{1} C_{u} S}{N}\left(1-\frac{A^{*}}{A}\right)\left(1-\frac{C_{u *} S * \mathrm{AN}}{C_{u} S A * N *}\right) \\
&+\frac{\beta \alpha_{2} C_{a} S}{N}\left(1-\frac{A^{*}}{A}\right)\left(1-\frac{C_{a *} S * \mathrm{AN}}{C_{a} S A * N *}\right)+\sigma A\left(1-\frac{C_{u}}{C_{u}}\right)\left(1-\frac{A^{*} C_{u}}{A C_{u} *}\right) \\
&+v_{2} C_{u}\left(1-\frac{C_{a}^{*}}{C_{a}}\right)\left(1-\frac{C_{u}^{*} C_{a}}{C_{u} C_{a} *}\right)+v_{1} A\left(1-\frac{C_{a}^{*}}{C_{a}}\right)\left(1-\frac{A^{*} C_{a}}{A C_{a} *}\right) \\
&+\delta C_{a}\left(1-\frac{T_{c}^{*}}{T_{c}}\right)\left(1-\frac{C_{a} * T_{c}}{C_{a} T_{c} *}\right)
\end{aligned}
$$

$P_{1} \leq 0$ whenever 
and $P_{2} \leq 0$ whenever

$S^{*} N \geq S N *, C_{u} * S * A N \geq C_{u} S A * N *, C_{a}{ }^{*} S^{*} A N \geq C_{a} S A * N *, A * C_{u} \geq A C_{u} *, C_{u} * C_{a} \geq C_{u} C_{a}{ }^{*}, A * C_{a} \geq A C_{a}{ }^{*}, C_{a}{ }^{*} T_{c} \geq C_{a} T_{c} *$

Thus, $\frac{d L}{d t} \leq 0$ if the condition in (35) and (36) holds.

therefore, by LaSalle asymptotic stability theorem (LaSalle, 1976), and Oke et al. (2020) the positive equilibrium state $\frac{d L}{d t}$ is globally asymptotically stable in the positive region $R_{+}^{6}$.

\section{Numerical computation}

The numerical study is carried out using maple software embedded code for the Runge-Kutta of fourth order. Here, the subsequent default values are assumed for the embedded parameters taken from theoretical studies in literatures $\gamma=$ $0.9, \beta=0.008, \sigma=0.59, d(c)=0.00693, \mu=0.00693, \omega=0.1, v_{1}=0.002, v_{2}=0.002, \alpha_{1}=0.0016, \alpha_{2}=0.0016, \delta=$ $0.0085, \Pi=0.07$. The values remain unchanged throughout* the computations except otherwise indicated.

The effects of varying the testing rate of the acute individuals $\left(v_{1}\right)$, testing rate of chronic individuals $\left(v_{2}\right)$ and treatment rate of chronic individuals $(\delta)$ on the population dynamics are shown in Figures 2 to 7 . From Figures 2 and 3 , an increase in the parameters values reduces susceptible and acute populations thereby reducing the spread HBV due to low interaction between the host immune system and the virus. Therefore, the appearance of HBV and the pathogenesis reduces, which in so doing, lessens the potential injury on the liver. Hence, the liver is shielded from hepatocellular carcinoma over time. The rate of chronic unaware and chronically aware individuals is examined in Figures 4 and 5. The parameter variations show a significant decrease in the chronic unaware population which implies that testing at that stage is a great tool for reducing the disease transmission. The transmission process dies down as the time progresses; this discourages liver inflammation as a result of lowering the infected individuals. Meanwhile, the chronic population in Figure 5, depicts a high significant influence of the acutely infected and chronically unaware infected individuals over

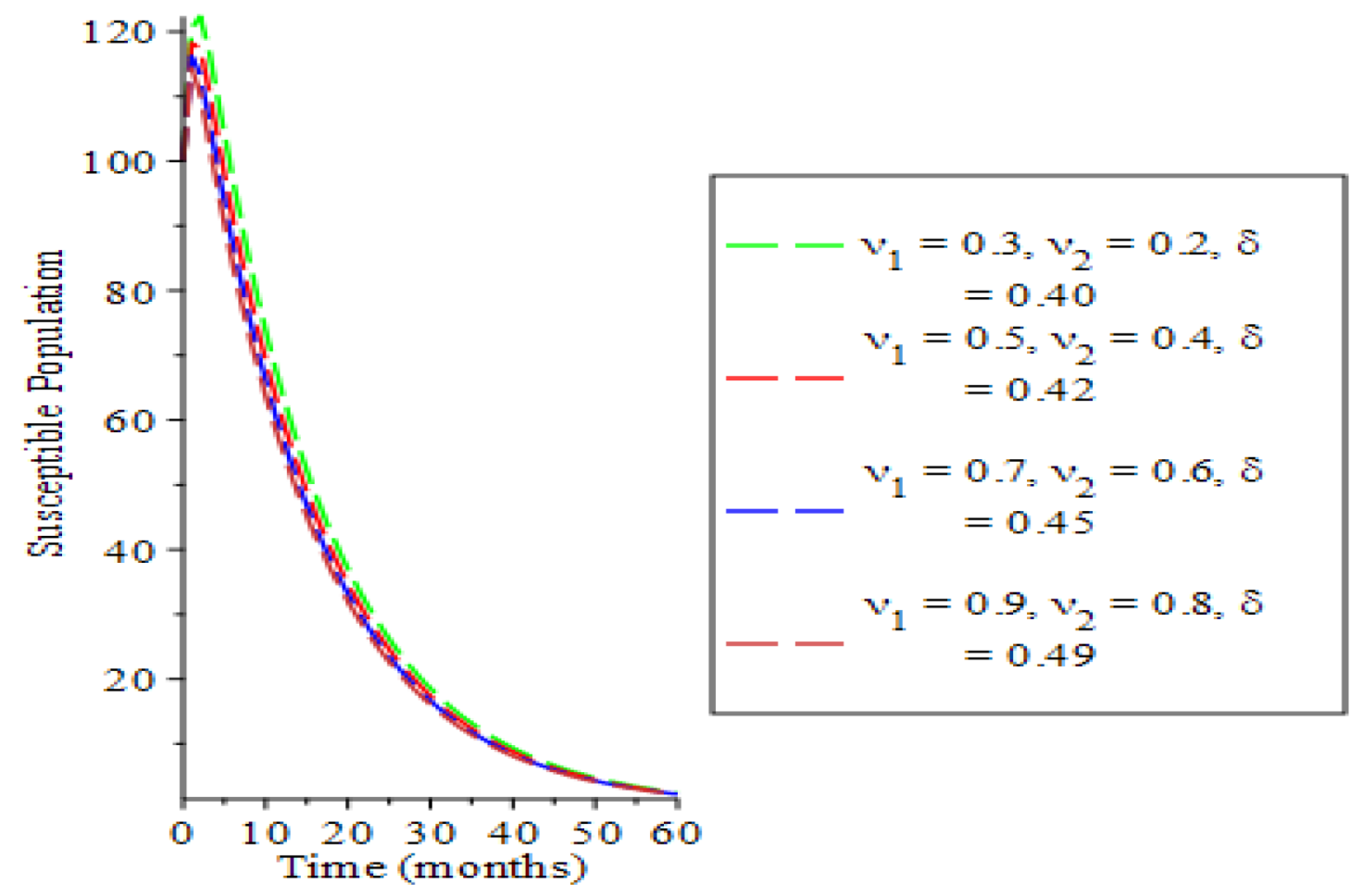

Figure 2. Behavioral dynamics of susceptible population when varying testing rate for acute and chronic individuals and treatment for chronic individuals. 


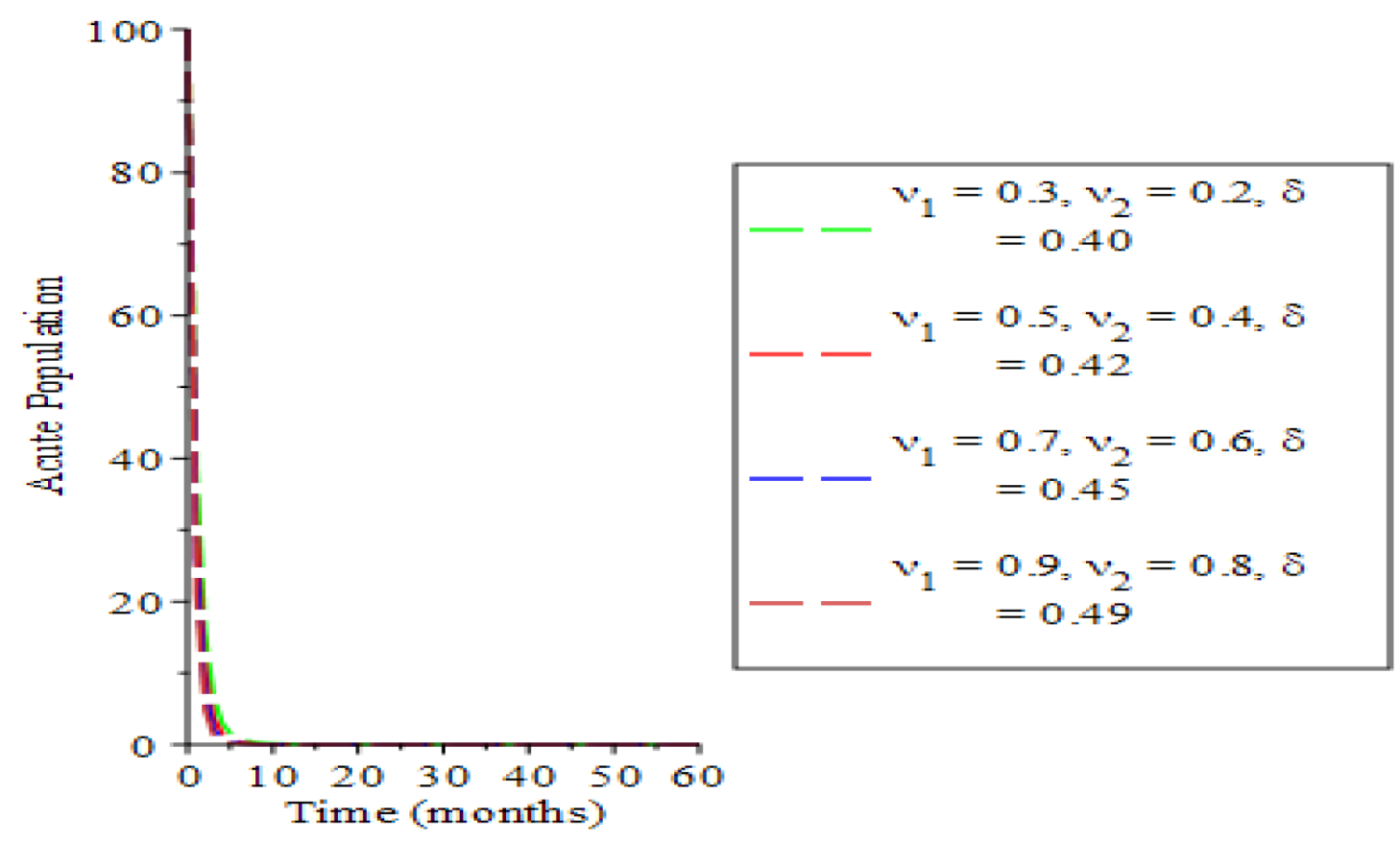

Figure 3. Behavioral dynamics of acute population when varying testing rate for acute and chronic individuals and treatment for chronic individuals.

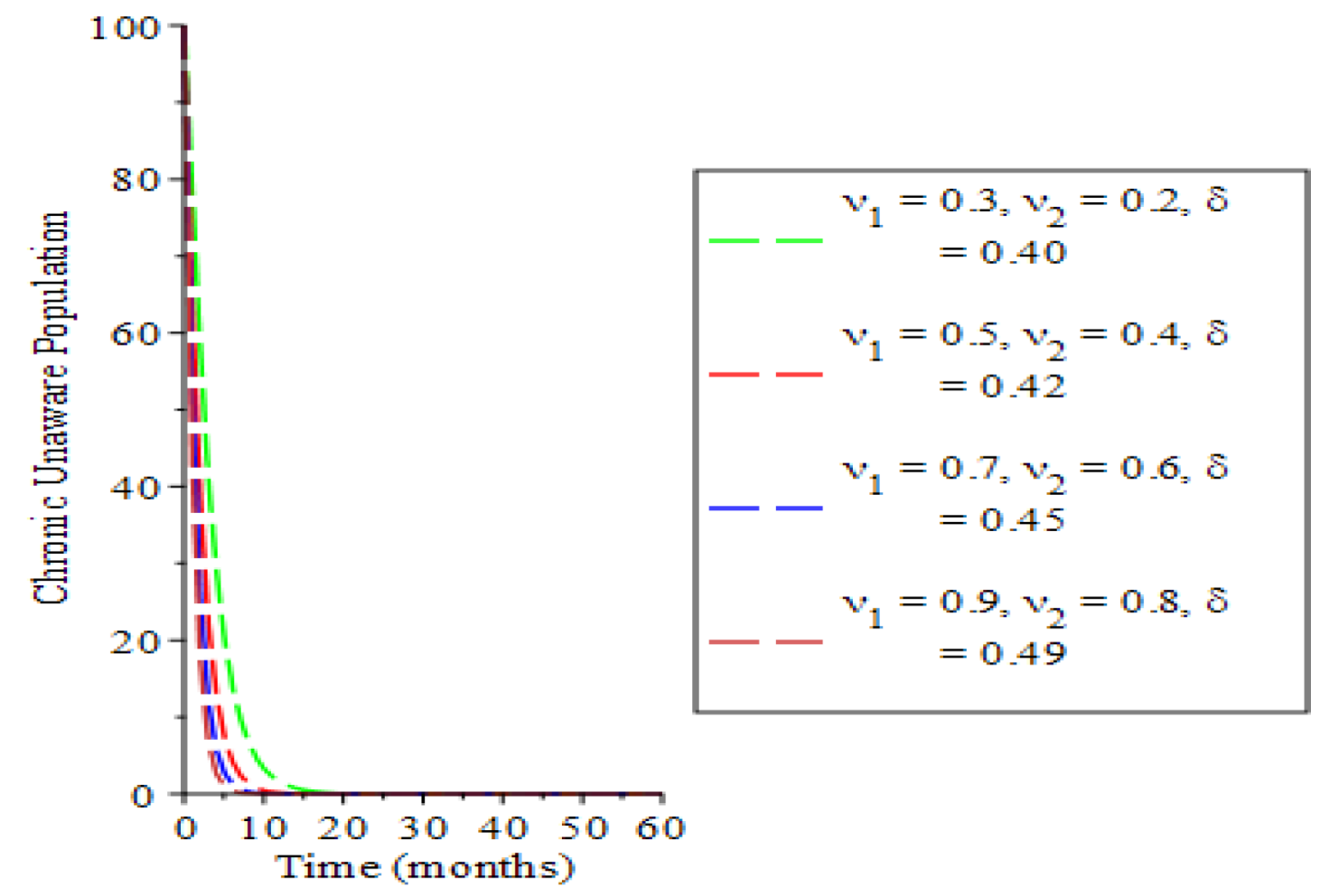

Figure 4. Behavioral dynamics of chronic unaware population when varying testing rate for acute and chronic individuals and treatment for chronic individuals. 


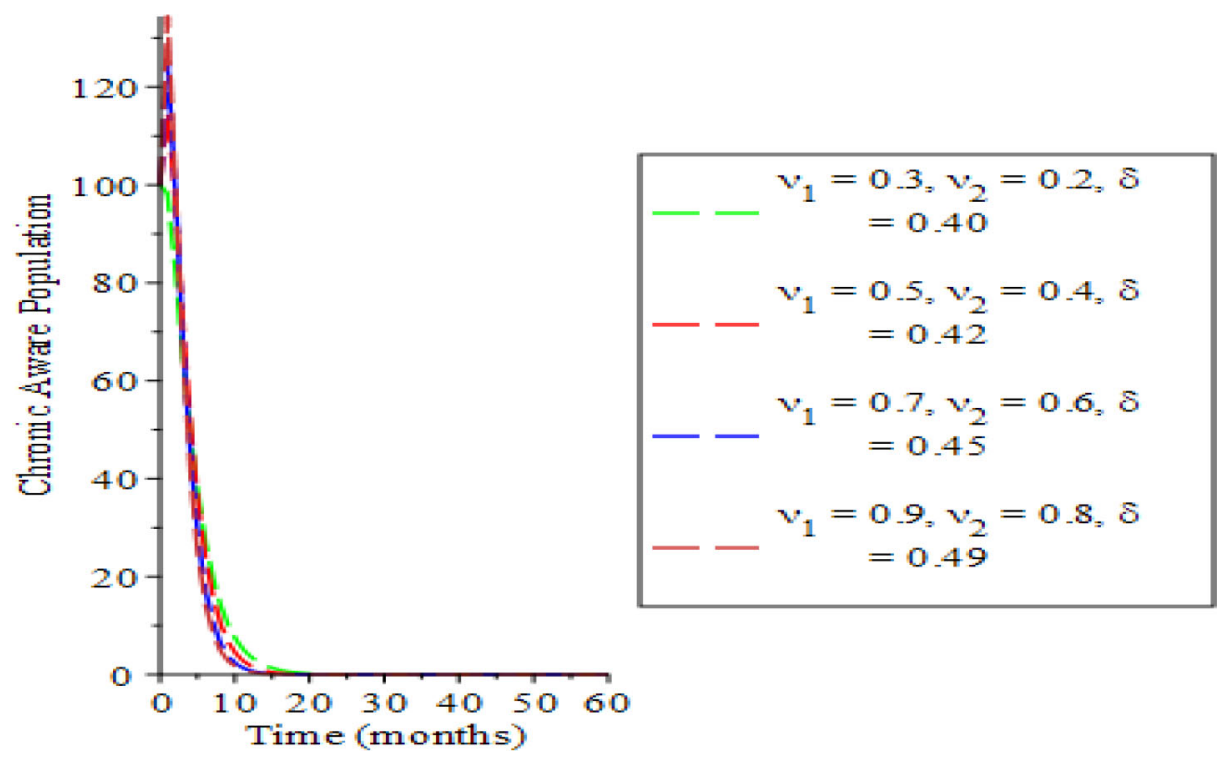

Figure 5. Behavioral dynamics of chronic aware population when varying testing rate for acute and chronic individuals and treatment for chronic individuals.

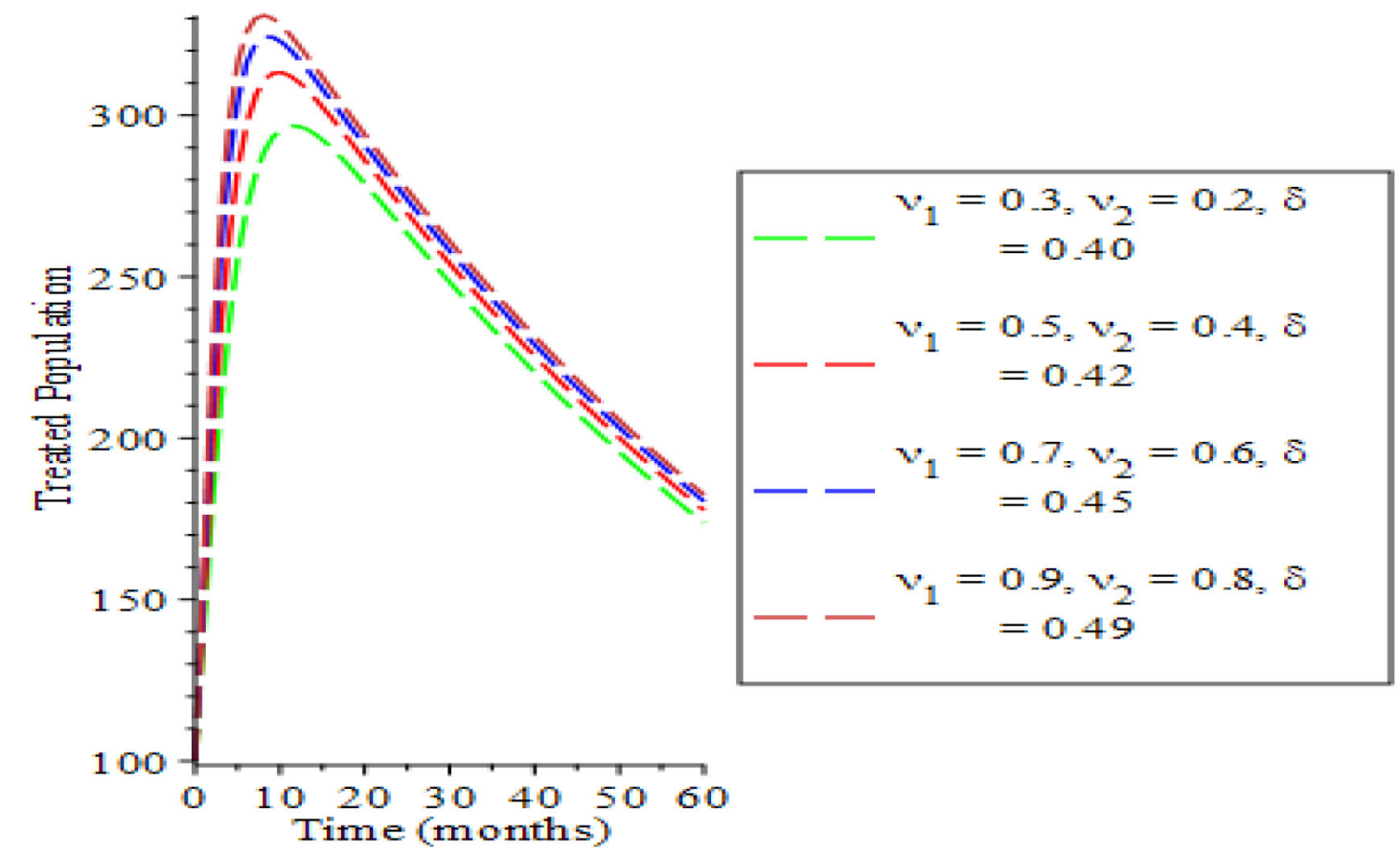

Figure 6. Behavioral dynamics of treated population when varying testing rate for acute and chronic individuals and treatment for chronic individuals.

time. A chronic infection phase is found at the time range $10<t<20$, as such, the individuals are exposed to liver carcinoma or cirrhosis. Hence, the chronic population diminishes as the parameters are increased.

In Figures 6 and 7, the impact of varying the testing rate of the acute individuals $\left(v_{1}\right)$, testing rate of chronic individuals $\left(v_{2}\right)$ and treatment rate of chronic individuals $(\delta)$ on the treated and the recovered population are presented. The treated population increases with parameters variation along the rising time $(t)$ as a result of long time effect of parameter values. The recovery rate is enhanced as observed in Figure 7 due to significant simulation of surface antibodies of Hepatitis B. 


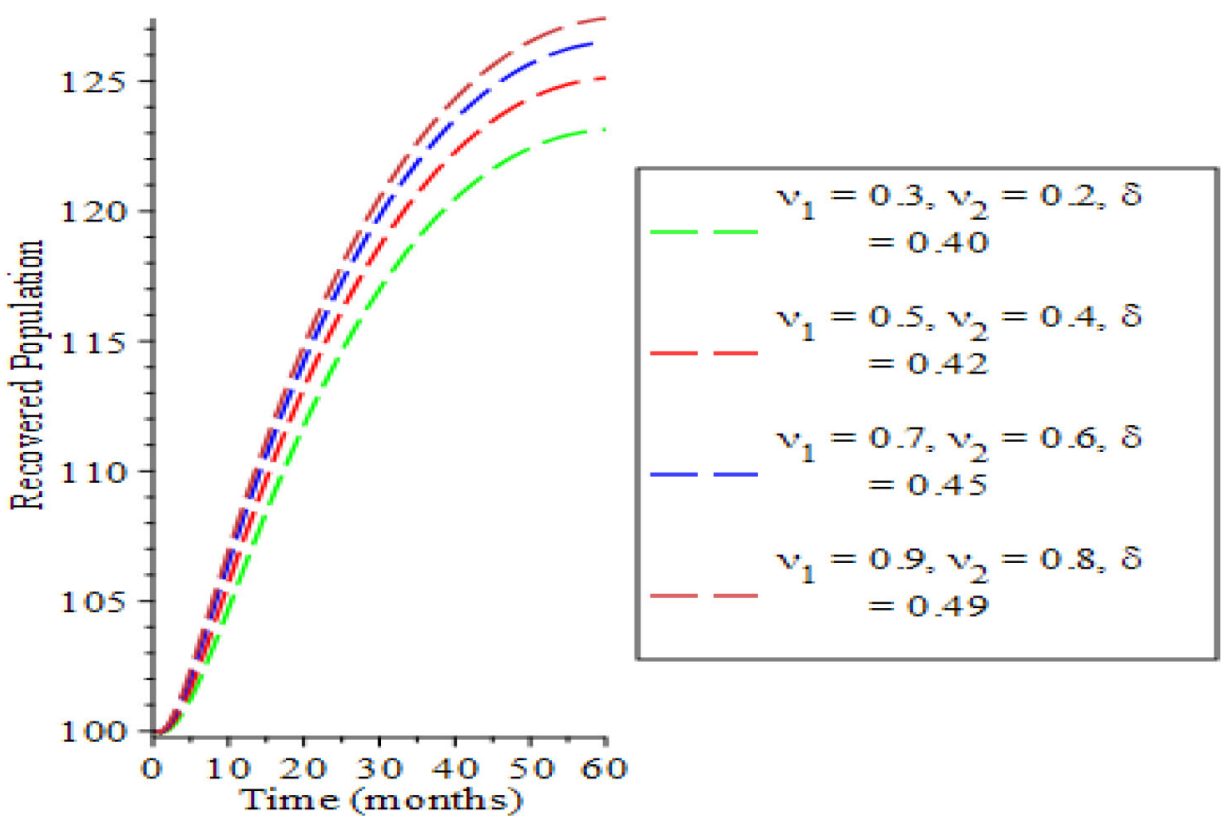

Figure 7. Behavioral dynamics of recovered population when varying testing rate for acute and chronic individuals and treatment for chronic individuals.

This is in conformity with the works of Pang et al. (2010) and Ullah et al. (2019). This result implies that an intensification in testing at all infectious states and rise in treatment of chronic individual will bring about a reduction in the HBV transmission process which is a response to the WHO goal for 2030 that concentrating efforts on awareness program and campaign will sure bring about a decrease or eradication in the transmission process of the virus (WHO, 2020).

\section{Conclusion}

A deterministic model of hepatitis B testing was developed and investigated, which included testing in the chronic unaware state as well as testing in all infectious states. The model has disease-free and endemic equilibria. The basic reproduction number was calculated using the next generation matrix method. The equilibria's local and global stability were discussed and shown to be asymptotically stable. The testing and treatment rate effects were thoroughly discussed.

\section{Data availability}

No data are associated with this article.

\section{Grant information}

None declared.

\section{Competing interests}

None declared.

\section{References}

Ciupe SM, Ribeiro RM, Perelson AS: Antibody Responses during Hepatitis B Viral Infection. PLoS Comput Biol. 2014; 10(7): e1003730.

PubMed Abstract | Publisher Full Text | Free Full Text

Cohen C, Holmberg SD, McMahon BJ, et al.: Is chronic hepatitis B being undertreated in the United States? J Viral Hepatol. 2011; 18(1): 377-383.

PubMed Abstract | Publisher Full Text

LaSalle JP: The Stability of Dynamical Systems, Regional Conference Series in Applied Mathematics Philadelphia, Pa,USA: SIAM; 1976.
Lin SY, Chang ET, So SK: Stopping a silent killer in the underserved Asian and pacific islander community: a chronic hepatitis B and liver cancer prevention clinic by medical students. Asian Pac J Cancer Prev. 2009; 10(9): 383-386.

PubMed Abstract

McPherson S, Valappil M, Moses SE, et al.: Targeted case finding for hepatitis B using dry blood spot testing in the British-Chinese and South Asian populations of the North-East of England.J Viral Hepatol. 2013; 20(1): 638-644.

PubMed Abstract | Publisher Full Text 
Meffre C, Le Strat Y, Delarocque-Astagneau E, et al.: Prevalence

of hepatitis $B$ and hepatitis $C$ virus infections in France in 2004: social factors are important predictors after adjusting for known risk

factors. J Med Virol. 2010; 82(2), 546-555.

PubMed Abstract | Publisher Full Text

Mpeshe SC, Nyerere N: Modeling approach to assess the transmission dynamics of Hepatitis B infection in Africa. Int J Advance App Math Mechanic. 2019; 6(3): 51-61.

Niederau C: Chronic hepatitis B in 2014: great therapeutic progress, large diagnostic deficit. World J Gastroenterol. 2014; 20(33): 11595-11617.

PubMed Abstract | Publisher Full Text | Free Full Text

Oke IS, Ojo MM, Adeniyi OM, et al.: Mathematical modeling of malaria disease with control strategy. Comm Math Biol Neurosci. 2020; 43(1)

180-188.

Publisher Full Text
Pang J, Cui JA, Zhou X: Dynamical behavior of a hepatitis B virus transmission model with vaccination. J Theoretical Biol. 2010; 265(4): 572-578.

PubMed Abstract | Publisher Full Text

Piorkowsky NY: Europe's hepatitis challenge: defusing the "viral time bomb". J Hepatol. 2009; 51(1): 1068-1073.

PubMed Abstract | Publisher Full Text

Ullah S, Khan MA, Gómez-Aguilar JF: Mathematical formulation of hepatitis B virus with optimal control analysis. Optim Control Appl Methods. 2019; 40(3): 529-544.

Publisher Full Text

Zhang J, Zhang S: Application and Optimal Control for an HBV

Model with Vaccination and Treatment. Discrete Dyn Nat Soc. 2018; 20(7):

69-73.

Publisher Full Text 


\section{Open Peer Review}

\section{Current Peer Review Status:}

\section{Version 1}

Reviewer Report 17 December 2021

https://doi.org/10.5256/f1000research.76473.r94650

(C) 2021 oke S. This is an open access peer review report distributed under the terms of the Creative Commons Attribution License, which permits unrestricted use, distribution, and reproduction in any medium, provided the original work is properly cited.

\section{Segun oke}

Department of Mathematical and Applied Mathematics, University of Pretoria, Pretoria, South Africa

The authors worked on the impact of testing and treatment on the dynamics of Hepatitis B virus. Although the manuscript is well presented and gives new insight into the Hepatitis B virus, especially for unvaccinated men who have sex with men (MSM) and heterosexual people who have multiple sexual partners or have contact with sex workers.

In their paper, a mathematical model was formulated to analyse the acquisition and transmission process of the virus with the view of identifying the possible way of reducing the menace and mitigating the risk of the virus. The models' positivity and boundedness were demonstrated using well-known theorems. Equating the differential equations to zero demonstrates the equilibria of the solutions i.e., the disease-free and endemic equilibrium. The next Generation Matrix method was used to compute the basic reproduction number for the models. Local and global stabilities of the models were shown via linearization and contrition of Lyapunov function methods respectively. The authors concluded by showing disease-free, endemic equilibria. The basic reproduction number was calculated using the next-generation matrix method. The equilibria's local and global stability were discussed and shown to be asymptotically stable. The testing and treatment rate effects were thoroughly discussed. I believe that I have an appropriate level of expertise to confirm that it is of an acceptable scientific standard.

\section{Some minor comments:}

1. The authors need to review more literature to back up their claims.

2. The introduction is too short and is not well cited. For example:

"In highly endemic areas, the most common form of transmission of hepatitis $B$ is from mother to child at birth (vertical transmission) or through horizontal route (contact with infected blood), particularly from infected children to uninfected children during the first five years of life. vaginal and spermatic fluids and, to a lesser degree, perspiration, breast milk, tears, and urine. In 
particular, hepatitis $B$ can be transmitted through sexual contact in unvaccinated men who have sex with men (MSM) and heterosexual people who have multiple sexual partners or have contact with sex workers" there is no citation here.

3. The authors need to add epidemiological interpretation and recommendations to the manuscript.

4. The conclusion needs to be rewritten.

Is the work clearly and accurately presented and does it cite the current literature? No

Is the study design appropriate and is the work technically sound?

Yes

Are sufficient details of methods and analysis provided to allow replication by others? Yes

If applicable, is the statistical analysis and its interpretation appropriate?

Not applicable

Are all the source data underlying the results available to ensure full reproducibility? Yes

Are the conclusions drawn adequately supported by the results? No

Competing Interests: No competing interests were disclosed.

Reviewer Expertise: Mathematical modelling, Mathematical Oncology, Optimal control, Epidemiology, etc

I confirm that I have read this submission and believe that I have an appropriate level of expertise to confirm that it is of an acceptable scientific standard.

Reviewer Report 06 October 2021

https://doi.org/10.5256/f1000research.76473.r94651

(c) 2021 Salawu S. This is an open access peer review report distributed under the terms of the Creative Commons Attribution License, which permits unrestricted use, distribution, and reproduction in any medium, provided the original work is properly cited.

\section{Sulyman Olakunle Salawu}

Department of Mathematics, Landmark University, Omu-aran, Nigeria 
Reviewer Reports on the impact of testing and treatment on the dynamics of Hepatitis B virus. The impacts of different parameters were examined. The manuscript is well written and presented logically. The abstract captures accurately the whole work and the introduction is sound. However, I have the following comments and queries for the authors:

1. The authors should explain what prompted the choice of method of solution used over other techniques.

2. The significance and motivation of the study are not clear. The authors should clarify these.

3. There is a need to reference equation (3) to establish its correctness and source.

4. Some grammatical mistakes should be checked through.

5. Why have the authors chosen to engage in this study?

6. A likely extension of the study should be stated or recommended.

The manuscript should be indexed subject to minor correction.

Is the work clearly and accurately presented and does it cite the current literature? Yes

Is the study design appropriate and is the work technically sound?

Yes

Are sufficient details of methods and analysis provided to allow replication by others? Yes

If applicable, is the statistical analysis and its interpretation appropriate? Not applicable

Are all the source data underlying the results available to ensure full reproducibility? No source data required

Are the conclusions drawn adequately supported by the results?

Yes

Competing Interests: No competing interests were disclosed.

Reviewer Expertise: Mathematical Modeling and flow dynamic

I confirm that I have read this submission and believe that I have an appropriate level of expertise to confirm that it is of an acceptable scientific standard. 
The benefits of publishing with F1000Research:

- Your article is published within days, with no editorial bias

- You can publish traditional articles, null/negative results, case reports, data notes and more

- The peer review process is transparent and collaborative

- Your article is indexed in PubMed after passing peer review

- Dedicated customer support at every stage

For pre-submission enquiries, contact research@f1000.com 